\title{
STUDIES ON SOLID DISPERSIONS OF LEFLUNOMIDE
}

\author{
MAHAPARALE PR ${ }^{1 *}$, THORAT VP² \\ ${ }^{1}$ Department of Pharmaceutics, Government College of Pharmacy, Aurangabad, Maharashtra, India. ${ }^{2}$ Department of Pharmaceutics, \\ Siddhi College of Pharmacy, Chikhali, Pune, Maharashtra, India. Email: pareshmahaparale@gmail.com
}

Received: 17 December 2018, Revised: 31 January 2020 Accepted: 11 April 2020

ABSTRACT

Objective: Leflunomide is a nonsteroidal anti-inflammatory drug, which is poorly water soluble. In the present study, attempt has been made to prepare and characterize solid dispersions of leflunomide to increase the solubility of drug.

Methods: In preparation of solid dispersion of leflunomide, different polymers such as polyethylene glycol (PEG) 4000, PEG 6000, Poloxamer 188, and Poloxamer 407 were used. The effects of several variables such as type of carrier used, drug: carrier ratios, and method of preparation were studied. The evaluation of solid dispersions was done by solubility study, dissolution study, and X-ray diffractometry.

Results: Improvement in dissolution of the drug was observed in all solid dispersions as compared to pure drug alone. Solid dispersions prepared using Poloxamer 188 showed fastest in vitro drug release. Solid dispersions prepared using solvent evaporation method showed relatively faster drug release than melt evaporation method. X-ray diffraction patterns indicated reduced crystallinity of drug particles, which suggest a mechanism of enhanced solubility and dissolution of drug in solid dispersion systems.

Conclusions: The study indicated that solid dispersion by solvent evaporation can successfully be further explored and employed to improve solubility and dissolution characteristics of poorly soluble drugs.

Keywords: Leflunomide, Solid dispersion, Carrier.

(c) 2020 The Authors. Published by Innovare Academic Sciences Pvt Ltd. This is an open access article under the CC BY license (http://creativecommons. org/licenses/by/4. 0/) DOI: http://dx.doi.org/10.22159/ajpcr.2020.v13i6.31283

\section{INTRODUCTION}

The recent advent of high throughput screening of potential therapeutic agents has given rise to number of poorly soluble drug candidates. The formulation of poorly soluble compounds in oral delivery presents one of the most frequent and greatest challenges to formulation scientists in the pharmaceutical industry. Drugs with low aqueous solubility have low dissolution rates and suffer from oral bioavailability problems. Many methods such as solubilization in surfactant systems, formation of water soluble complexes, use of prodrug, and salt formation approach have been reported for increasing solubility, dissolution, and, in turn, bioavailability of drugs. The solid dispersion is defined as dispersion of one or more active ingredients in an inert carrier matrix at solid state prepared by the melting (fusion), solvent evaporation, or meltingsolvent method [1-3]. Leflunomide is a nonsteroidal anti-inflammatory drug, which is poorly water soluble.

\section{MATERIALS AND METHODS}

\section{Materials}

Leflunomide (Alembic Pharmaceuticals Ltd., Baroda) and various carriers such as polyethylene glycol (PEG) 4000, PEG 6000 (Qualigens Fine Chemicals, Mumbai), Poloxamer 188, and Poloxamer 407 (BASF, Germany) were used. All other chemicals and reagents were of analytical grade.

\section{Methods}

Preparation of solid dispersions

Solid dispersions of leflunomide were prepared by the following two methods.

\section{Solvent evaporation method}

Leflunomide and each of water-soluble carriers such as PEG 4000, PEG-6000, Poloxamer-188, and Poloxamer-407 were accurately weighed separately, transferred into a beaker, and dissolved in a sufficient quantity of methanol. Subsequently, methanol was evaporated in vacuum evaporator and resulting solid dispersions were stored for $24 \mathrm{~h}$ in a desiccator to remove traces of solvent. Finally, the resultant mass was triturated in glass mortar and passed through sieve no. 44. The resulting solid dispersions were stored in tightly-closed containers until further use $[4,11]$

\section{Melt evaporation method}

Leflunomide and each of water-soluble carriers such as PEG 4000, PEG-6000, Poloxamer-188, and Poloxamer-407 were accurately weighed separately in porcelain dish and heated to melt. The drug was added to a molten mass of polymers with vigorous stirring. Resulting solid dispersions were stored in a desiccator for $24 \mathrm{~h}$. The resultant mass was triturated in a glass mortar and passed through sieve no. 44. The resulting solid dispersions were stored in tight-closed containers until further use $[4,5]$.

\section{Evaluation of solid dispersions}

Drug content estimation

The percentage drug content in solid dispersions was estimated by dissolving quantities of solid dispersions equivalent to $10 \mathrm{mg}$ of leflunomide in $100 \mathrm{ml}$ of methanol. These solutions were further diluted with distilled water and UV absorbance was recorded at $259.5 \mathrm{~nm}[6,7]$.

Saturation solubility study

Solubility study was performed according to the method reported by Higuchi and Connors [3].

\section{$X$-ray diffraction (XRD) study of solid dispersions}

XRD patterns of the selected solid dispersions were compared with that of the plain leflunomide. This was done by measuring the $2 \varnothing$ in the range of $4-50^{\circ}$ with reproducibility of $\pm 0.001^{\circ}$ on X-ray diffractometer (Philips). The XRD patterns were recorded automatically using rate meter with time constant of $2 \times 10^{2}$ pulse/s and with the scanning speed of $2^{\circ}(2 \varnothing) / \mathrm{min}$. 
Table 1: Comparison of various parameters for leflunomide solid dispersion systems

\begin{tabular}{|c|c|c|c|c|c|c|}
\hline \multirow[t]{2}{*}{ System } & \multicolumn{2}{|c|}{$\%$ drug content } & \multicolumn{2}{|c|}{ Solubility $(\mathrm{gm} / 100 \mathrm{ml})$} & \multicolumn{2}{|c|}{$\mathrm{T}_{90(\min )}$} \\
\hline & SE & ME & SE & ME & SE & ME \\
\hline LF (alone) & - & & 0.0024 & & $>90$ & \\
\hline LF:PEG 4000 (1:5) & $98.01 \pm 0.46$ & $96.96 \pm 0.46$ & 0.016 & 0.016 & $>90$ & $>90$ \\
\hline LF:PEG 6000 (1:3) & $96.38 \pm 0.65$ & $97.73 \pm 0.72$ & 0.015 & 0.012 & $>90$ & $>90$ \\
\hline LF:PEG 6000 (1:5) & $98.32 \pm 1.12$ & $96.89 \pm 0.64$ & 0.023 & 0.018 & 29 & 54 \\
\hline LF:P188 (1:3) & $95.52 \pm 1.65$ & $98.29 \pm 1.21$ & 0.032 & 0.028 & 29 & 54 \\
\hline LF:P188 (1:5) & $98.28 \pm 2.09$ & $98.80 \pm 1.21$ & 0.052 & 0.046 & 7 & 8 \\
\hline LF:P407 (1:3) & $96.69 \pm 2.09$ & $98.54 \pm 0.46$ & 0.024 & 0.021 & 57 & 90 \\
\hline LF:P407 (1:5) & $97.57 \pm 1.65$ & $98.12 \pm 1.21$ & 0.038 & 0.032 & 22 & 71 \\
\hline LF:PEG 4000 (1:5) PM & $96.52 \pm 1.88$ & $97.82 \pm 0.96$ & 0.0072 & 0.0065 & $>90$ & \\
\hline LF:PEG 6000 (1:5) PM & $98.32 \pm 1.92$ & $98.22 \pm 1.12$ & 0.01 & 0.0093 & $>90$ & \\
\hline LF:P407 (1:5) PM & $97.52 \pm 1.25$ & $96.95 \pm 1.40$ & 0.018 & 0.018 & $>90$ & \\
\hline LF:P188 (1:5) PM & $98.42 \pm 0.98$ & $97.25 \pm 1.02$ & $0.028 \pm 1.62$ & $0.025 \pm 1.08$ & 68 & \\
\hline
\end{tabular}

*LF: Leflunomide, P188: Poloxamer 188, P407: Poloxamer 407, SE: Solvent evaporation method, ME: Melt evaporation method, PM: Physical mixture

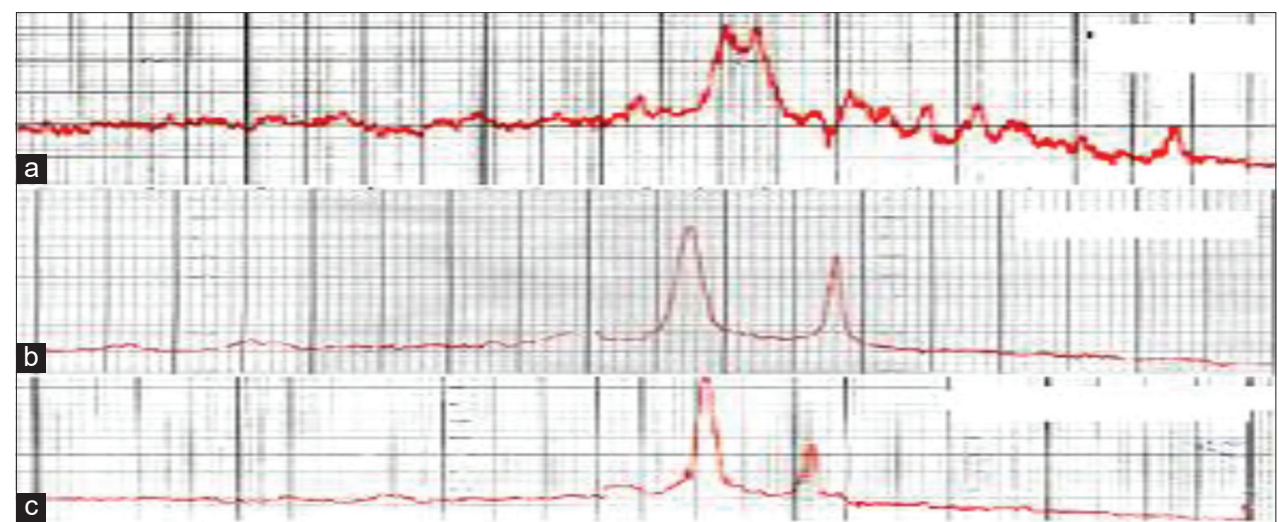

Fig. 1: X-ray diffraction pattern for a - leflunomide (pure), b - Poloxamer 188 (pure), and c - their solid dispersion prepared by solvent evaporation method

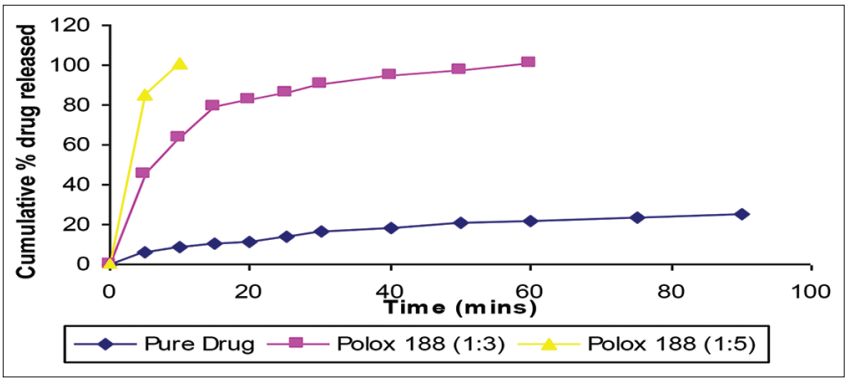

Fig. 2: Effect of carrier ratio on dissolution profile of leflunomide solid dispersion

Dissolution study of solid dispersions

Solid dispersions equivalent to $20 \mathrm{mg}$ of leflunomide were filled in hard gelatin capsule by the hand filling method. Dissolution study was carried out using USP XXII six-station programmable dissolution test Type II apparatus (Veego). Dissolution study was carried out in $900 \mathrm{ml}$ of $0.1 \mathrm{~N} \mathrm{HCl}$ at $37 \pm 0.5^{\circ} \mathrm{C}$ at $50 \mathrm{RPM}$. The $\mathrm{T}_{90}$ (time required for $90 \%$ dissolution of the drug) (Table 1) of various solid dispersions was calculated $[8-10,12]$.

\section{RESULTS AND DISCUSSION}

\section{Evaluation of solid dispersions}

Drug content estimation

The percentage drug content of all solid dispersions of leflunomide was between $95.52 \%$ and $99.86 \%$ (Table 1). Standard deviation " $<3$ " indicates the uniform distribution of drugs in various solid dispersions.

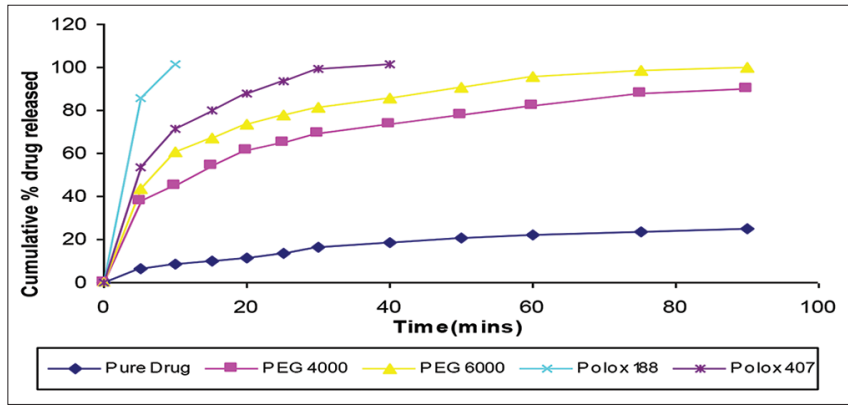

Fig. 3: Effect of carrier type on dissolution profile of leflunomide solid dispersion

Saturation solubility study

All solid dispersions showed enhancement in the solubility as compared to pure drug alone (Table 1). The drug: carrier ratio (1:5) showed higher solubility in all solid dispersions. This may be due to the higher proportion of hydrophilic carriers present in solid dispersions. The drug gets uniformly dispersed in water-soluble carriers. Hence, as soluble carrier dissolves, the dispersed drug also gets exposed to an aqueous environment as very fine particles and solubility gets increased. Enhancement in solubility was observed in the following order,

\section{Poloxamer 188>Poloxamer 407>PEG 6000>PEG 4000 .}

XRD study of solid dispersions

Dissolution properties of drug particles are greatly affected by the extent of crystallinity present in solid dispersion. An amorphous or the 


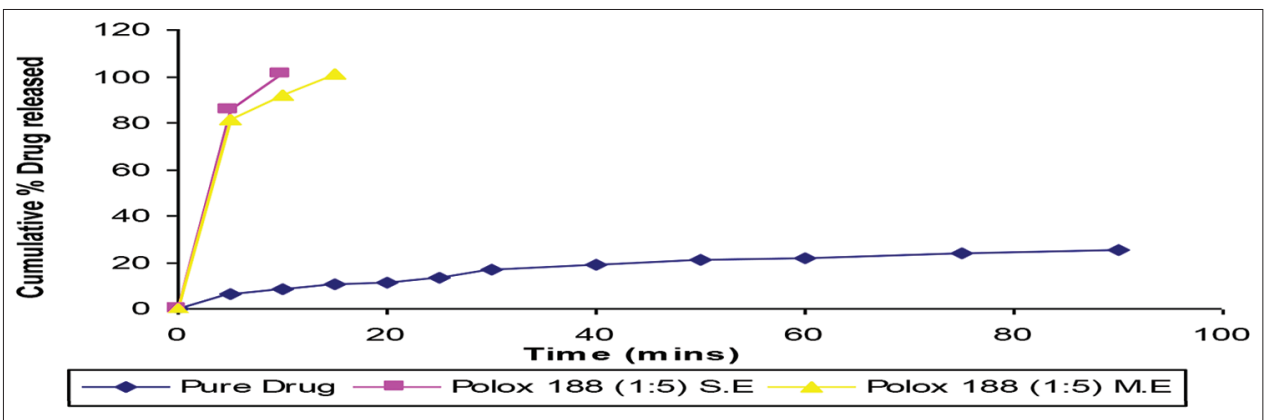

Fig. 4: Effect of preparation method on dissolution profile of leflunomide solid dispersion

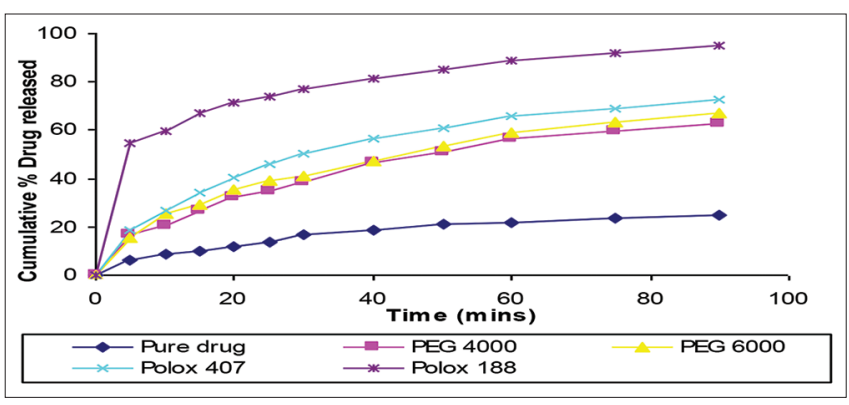

Fig. 5: Effect of carrier type on dissolution rate of leflunomide from physical mixture

metastable form dissolves faster because of associated higher levels of internal energy and greater molecular mobility. These together enhance the thermodynamic properties of these forms as compared to crystalline state. XRD studies were performed to reveal the crystallinity of pure leflunomide, its dispersion with Poloxamer 188 (1:5) and pure Poloxamer188 (Fig. 1). Many broad peaks of low intensity were observed in diffraction patterns, which indicate microcrystalline nature of the drug. The XRD pattern of Poloxamer 188 showed diffraction peaks of very low intensity, which confirmed the amorphous nature. The XRD pattern for solid dispersions prepared with Poloxamer 188 (1:5), however, was characterized by the absence of diffraction peaks of the drug, suggesting conversion of microcrystalline form of the drug to an amorphous state [17-20]

\section{Dissolution study of solid dispersions}

Solid dispersions of drugs showed better dissolution performance as compared to pure drug alone. After $1.5 \mathrm{~h}$, release of the drug was found to be $25.01 \%$. All solid dispersions of drug showed $68-100 \%$ release of drug in $<1.5 \mathrm{~h}$. This may be attributed to improved wettability of the drug particles, significant reduction in particle size of the drug during formation of solid dispersions, and higher intrinsic dissolution rate of selected soluble carriers, which can pull insoluble but finely mixed drug particles into the bulk of the dissolution medium [14-16,21].

Effect of carrier ratio on dissolution rate of leflunomide from different solid dispersions

Two different drug: carrier ratios (1:3 and 1:5) were selected to assess the effect of the weight fraction of carrier on the release profile of drug from solid dispersions (Fig. 2). The dissolution of drug from solid dispersions prepared using a higher weight fraction of carrier was found to be more; this may be attributed to the molecular and colloidal dispersion of the drug in those carrier matrixes.

Effect of carrier type on dissolution rate of leflunomide from different solid dispersions

Solid dispersions prepared by both solvent evaporation and melt evaporation method showed improved dissolution profiles of a drug (Fig. 3). Enhancement in the dissolution profile of the drug was found to be of Poloxamer 188>Poloxamer 407>PEG 6000>PEG 4000. The

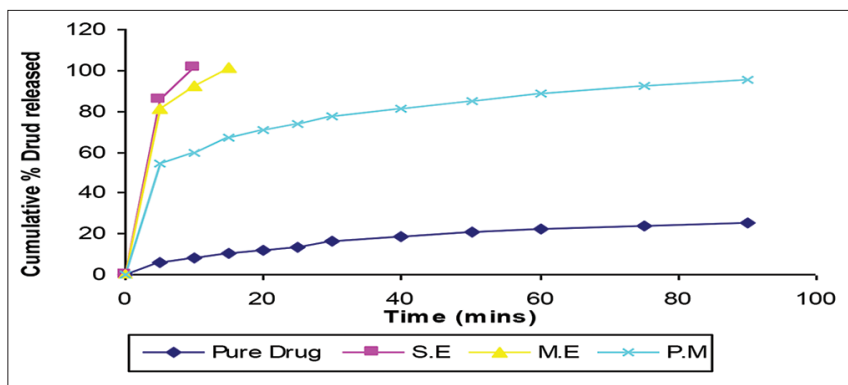

Fig. 6: Comparative dissolution profile of leflunomide from solid dispersion and physical mixture

increase in dissolution of solid dispersion with Poloxamer 188 may be due to high HLB value (HLB 29). Poloxamer 407 HLB value is 21.

Effect of preparation method on dissolution profiles of leflunomide from different solid dispersions

Solid dispersions prepared by solvent evaporation method showed slightly faster drug release as compared to solid dispersions obtained with the melted evaporation method (Fig. 4). In solvent evaporation method, both drug and polymer are dissolved in a common solvent because of that both may be present in molecular state. When solvent is evaporated, drug may get molecularly dispersed in hydrophilic carrier matrix. In melt evaporation method, solution of drug is added with stirring to melt mass of polymer, which is at a higher temperature than drug solution. At that temperature, solvent gets evaporated out and some of the drug may precipitate out, and hence, total molecular dispersion may not be achieved as that of solvent evaporation method.

Effect of carrier type on dissolution rate of leflunomide from physical mixture

Dissolution of drugs from all physical mixtures was found to be more as compared to pure drug (Fig. 5). This may be due to hydrophilicity of carriers. Dissolution of drug from solid dispersion was found to be more than from physical mixture (Fig. 6). This may be due to molecular dispersion of the drug in hydrophilic carriers in solid dispersion than physical dispersion only.

\section{CONCLUSIONS}

From the study on solid dispersions of leflunomide, it was found that,

- Enhancement in solubility and dissolution of solid dispersions is in the following order, Poloxamer 188>Poloxamer 407>PEG 6000>PEG 4000

- Solid dispersions prepared by both solvent evaporation and melt evaporation method showed significant improvement in dissolution profiles

- The dissolution rate for all solid dispersions increased with increasing concentration of carriers

- Method of preparation of solid dispersions affected the dissolution profile of leflunomide. For the same carrier employed, solid dispersions prepared by solvent evaporation showed more dissolution than those prepared by melting evaporation method 
- The enhanced dissolution rate with Poloxamer may be attributed to surfactant activity, which facilitates wetting and subsequent solubilization of the drug. Poloxamer 188 has high HLB value (HLB 29). Hence, it has a greater tendency to solubilize in water.

\section{ACKNOWLEDGMENT}

Authors are thankful to Alembic Pharmaceuticals Ltd., Baroda, for providing a gift sample of the drug. The authors are thankful to Principal, Dr. D Y Patil College of Pharmacy, Akurdi, Pune, for availing the facility to carry out the research work.

\section{CONFLICTS OF INTEREST}

The authors declare no conflicts of interest.

\section{REFERENCES}

1. Shah H. Dissolution improvement of nebivolol hydrochloride using solid dispersion adsorbate technique. Asian J Pharm 2015;9:49-55.

2. Serajuddin AT. Solid dispersion of poorly water soluble drugs: Early promises, subsequent problems and recent breakthroughs. J Pharm Sci 1999;88:1058-66.

3. Higuchi T, Connors KA. Phase solubility techniques. Adv Anal Chem Instrum 1965;4:117-212.

4. Mahaparale PR, Malpure PS, Sanap D, Kachare VT, Kasture PV. Development of dissolution medium of leflunomide. Indian Pharm 2007;6:69-70.

5. Batra V, Shirolkar SV, Mahaparale PR, Kasture PV. Solubility and dissolution enhancement of glipizide by solid dispersion technique. Indian J Pharm Educ Res 2008;42:373-8.

6. Deshpande KB, Ganesh NS. Orodispersible tablets-an overview of formulation and technology. Int J Pharm Bio Sci 2011;2:531-9.

7. Ratnakar R, Goswami L. Formulation and evaluation of fast dissolving tablet of telmisartan. Int J Pharm Chem Sci 2013;2:2078-85.

8. Mehta A, Tyagi R, Shukla A. Formulation and evaluation of solid dispersion of an antidiabetic drug. Curr Trends Biotech Pharm 2009;3:76-84

9. Amidon GL, Lennernas H, Shah VP, Crisen JR. A theoretical basis for a biopharmaceutics drug classification: The correlation of in vitro drug products dissolution and in vivo bioavailability. Pharm Res 1995; $12: 413-24$

10. Somasundaram J, Mekonnen T. Formulation and evaluation of diltiazem hydrochloride oral dispersible tablets. Int J Pharm Health Care Res 2013;1:184-90.

11. Arora SC, Sharma PK, Irchhaiya R, Khatkar A, Singh N, Gagoria J. Development, characterization and solubility study of solid dispersions of azithromycin dihydrate by solvent evaporation method. J Adv Pharm Technol Res 2010;1:221-8.

12. Pekamwar SS. Formulation and evaluation of solid dispersion of lopinavir by using different techniques. Int Res J Pharm 2015;6:663-9.

13. Deshkar SS, Sonkamble K, Mahore J. Formulation and optimization of nanosuspension for improving solubility and dissolution of gemfibrozil. Asian J Pharm Clin Res 2019;12:157-63.

14. Yadav S, Veena M, Sriniva M. Solid dispersion technique to enhance the solubility and dissolution rate of aripiprazole by fusion method. Int J Pharm Pharm Sci 2016;8:187-92.

15. Shah I, Bhatt S, Yadav A. Enhancement of solubility and dissolution of nebivolol by solid dispersion technique. Int $\mathrm{J}$ Pharm Pharm Sci 2014;6:566-71.

16. Mohan A, Sangeetha G. In vitro-in vivo evaluation of fast dissolving tablets containing solid dispersion of oxcarbazepine. Int J Pharm Pharm Sci 2016;8:124-31

17. Mannem V, Suryadevara V. Formulation and evaluation of telmisartan solid dispersion using Entada scandens seed starch and poloxamer 188 as superdisintegrantss. Asian J Pharm Clin Res 2018;11:474-81.

18. Manimaran V, Dhamodharan N. Devlopment of fast dissolving tablets of amlodipine besylate by solid dispersion technology using poloxamer 407 and poloxamer 188. Asian J Pharm Clin Res 2017;10:135-41.

19. Singh G, Singh N. Development and characterization of nevirapine loaded amorphous solid dispersions for solubility enhancement. Asian J Pharm Clin Res 2019;12:176-82.

20. Swain RP, Subudhi BB. Amorphous solid dispersions of pioglitazone hydrochloride using cremophor RH 40 and poloxamer 188: In vitro and in vivo evaluation. Indian Drug 2019;56:45-55.

21. Jadhav P, Yadav A. Formulation, optimization, and in vitro evaluation of polymeric nanosuspension of flurbiprofen. Asian J Pharm Clin Res 2019;12:183-91. 